\section{ARTIGO ORIGINAL ORIGINAL ARTICLE}

\title{
Indicadores para avaliação econômica da aquisição hospitalar de medicamentos
}

\author{
Indicators for economic evaluation of drug hospital's acquisition \\ Patrícia de Carvalho Mastroianni', Alice Rosa Alves de Oliveira', \\ Tales Rubens de Nadai², Rosa Camila Lucchetta ${ }^{3}$ \\ DOI: 10.21115/JBES.v9.n2.p177-84
}

\section{RESUMO}

Objetivos: Propor indicadores de avaliação de aquisição de medicamentos e comparar os seus resultados econômicos. Métodos: Avaliou-se o resultado da compra de medicamentos do Hospital Estadual Américo Brasiliense (HEAB) por meio de um estudo descritivo e transversal. Para isso, foi realizada pesquisa documental dos relatórios de compra e de consumo de medicamentos por um ano. Obteve-se o número de medicamentos adquiridos em cada compra, seus preços unitários e a taxa de consumo mensal em unidades, para elaboração da curva $A B C$ e cálculo dos indicadores de percentual econômico - percentual de economia entre o preço máximo de venda ao governo (PMVG) 18\% CMED e preço médio unitário das aquisições analisadas - e razão econômica - razão entre o PMVG 18\% CMED e o preço médio unitário das aquisições analisadas. Resultados: Foi calculada a eficiência da aquisição para 51/72 apresentações classificadas como A e B, segundo curva ABC. A mediana da economia total gerada calculada pelo percentual econômico foi de 41,6\% (mínimo = -2008,6\%, máximo = 99,3\%, intervalo interquartil (IIQ 25-75\%) =-15,2-54,9) e de 0,6 (mínimo = 0, máximo $=21,1, \| \mathrm{Q} 25-75 \%=0,5-1,2$ ) calculada pela razão econômica. Conclusões: Observou-se que o processo de aquisição do HEAB foi eficiente, porque gerou economia. Devido à similaridade dos resultados obtidos pelos dois indicadores, consideram-se ambos elegíveis e aplicáveis para o monitoramento da economia gerada. No entanto, o indicador razão econômica seria o melhor aceito e implantável pelo serviço devido a maior facilidade de cálculo e interpretação do que o indicador percentual econômico.

\section{Keywords:}

drug price, drug costs, management indicators, economic indexes, benchmarking

\begin{abstract}
Objectives: To propose indicators of evaluation of drug acquisition and to compare their economic results. Methods: The results of the purchase of medications from the Hospital Estadual Américo Brasiliense (HEAB) were evaluated through a descriptive and cross-sectional study. To this end, a documentary survey of drugs purchases and consumption reports was accomplished for a year. The number of medicines purchased in each acquisition, their unit prices, and the monthly consumption rate in units were obtained in order to elaborate the $A B C$ analysis and to calculate the indicators of economic percentage - percentage of savings between the CMED maximum selling price to the government (MSPG) 18\% and average unit price of the analyzed acquisitions - and economic ratio - ratio between the CMED (MSPG) 18\% and the average unit price of the analyzed acquisi-
\end{abstract}

Recebido em: 20/03/2017. Aprovado para publicação em: 13/06/2017

1. Departamento de Fármacos e Medicamentos, Faculdade de Ciências Farmacêuticas, Universidade Estadual Paulista "Júlio de Mesquita Filho" (Unesp), Araraquara, SP, Brasil.

2. Hospital Estadual Américo Brasiliense (HEAB), Américo Brasiliense, São Paulo, SP, Brasil.

3. Programa de Pós-Graduação em Ciências Farmacêuticas, Universidade Federal do Paraná (UFPR), Curitiba, PR, Brasil. Instituição onde o trabalho foi executado: Hospital Estadual Américo Brasiliense (HEAB), São Paulo, SP, Brasil.

Financiamento: Pró-reitora de Extensão (PROEX) da Universidade Estadual Paulista "Júlio de Mesquita Filho" (Unesp) por meio de apoio financeiro e bolsa ao Projeto de Extensão em Farmacovigilância.

Agradecimentos: À Pró-reitora de Extensão (PROEX) pelo financiamento e à graduanda Beatriz Urias por auxiliar a coleta de dados, ao Diretor Administrativo do Hospital, Mário Sérgio de Bezerra Menezes, e a Supervisora Administrativa, Márcia Regina Batista Gürtler.

Congressos onde o estudo foi apresentado: Os resultados preliminares deste estudo foram apresentados em forma de pôster no XXVIII Congresso de Iniciação Científica da Universidade Estadual Paulista "Júlio de Mesquita Filho" (Unesp) (2016).

Autores correspondentes: Patrícia de Carvalho Mastroianni. Rodovia Araraquara-Jaú. Km 1, s/n, bairro Campus Ville, Araraquara SP, Brasil. CEP: 14800-903. Telefone: (+55 16) 3301-6977. E-mails: pmastro@fcfar.unesp.br, patriciamastroianni@yahoo.com.br Rosa Camila Lucchetta. Universidade Federal do Paraná, Curitiba, PR, Brasil. E-mail: rc.lucch@yahoo.com.br 
tions. Results: The acquisition efficiency was calculated for 51/72 presentations classified as A and B, according to the $\mathrm{ABC}$ curve. The total saving median engendered by the economic percentage was 41,6\% (minimum $=-2008,6 \%$, maximum $=99,3 \%$, interquartile range (IIQ 25-75\%) $=-15-54,9$ ) and 0.6 (minimum $=0$, maximum $=21.1, \| \mathrm{Q} 25-75 \%=0.5-1.2$ ) calculated by the economic ratio. Conclusions: It was observed that the acquisition process of HEAB was efficient, since it engendered savings. Because of the similarity of the results obtained by the two indicators, both are considered eligible and applicable to monitoring the economy. However, the economic ratio indicator would be better accepted and implantable by the service due to greater ease of calculation and interpretation than the economic percentage.

\section{Introdução}

A aquisição de medicamentos é uma das atividades do Ciclo da Assistência Farmacêutica que objetiva assegurar o abastecimento de medicamentos em quantidade adequada, qualidade assegurada, ao menor custo possível, dentro da realidade do mercado, promovendo o uso racional de medicamentos, em local e tempo determinados (Brasil, 1998). Essa premissa vai ao encontro da definição de uso racional de medicamentos estabelecida pela política nacional de medicamentos (Brasil, 1998) e a Organização Mundial de Saúde (Conferencia de Expertos, 1986): "Existe uso racional quando os pacientes recebem os medicamentos apropriados à sua condição clínica, em doses adequadas às suas necessidades individuais, por um período de tempo adequado e ao menor custo possível para eles e sua comunidade".

No serviço público, a aquisição de medicamentos deve considerar o menor preço e a melhor qualidade (Brasil, 1993; Brasil 2002). Além disso, é obrigatória a aquisição apenas de medicamentos registrados pelo Ministério da Saúde, no qual o processo de registro permite e garante no âmbito técnico, jurídico e administrativo a qualidade, a segurança e a eficácia do medicamento aprovado, dentre outros documentos necessários às empresas licitantes, que comprovem, por exemplo, a regularidade fiscal e a qualificação econômico-financeira da empresa (Brasil, 1993; Mastroianni e Lucchetta, 2011).

Logo, partindo do pressuposto de que todos os medicamentos registrados possuem qualidade comprovada, o critério determinante acaba sendo o preço, exceto nos casos em que há histórico de não conformidade do fornecedor ou notificações de inefetividade terapêutica, desvio de qualidade e queixa técnica do produto ou, ainda, outros dados de farmacovigilância que evidenciam falta de qualidade do produto pós-comercialização (Capucho, 2008; Capucho et al., 2008)

Um processo efetivo de aquisição de medicamentos deve ser feito de forma racional, baseado em evidência científica, a fim de garantir a otimização dos gastos públicos com saúde (Secoli et al., 2005). Nesse sentido, o uso de indicadores capazes de monitorar o desempenho das atividades da Farmácia Hospitalar, bem como a realização de estudos com a finalidade de aprimorar e/ou desenvolver indicadores mais apropriados para tal função, é essencial (Magarinos-Torres et al., 2007).
De acordo com Donabedian, uma avaliação deve ser facilmente mensurável e razoavelmente válida, compreendendo aspectos de estrutura, processo ou resultado (Donabedian, 2005). Os atributos de um indicador são: validade, sensibilidade, especificidade, simplicidade, objetividade e baixo custo (Bittar, 2001).

Neste contexto, propor indicadores qualificados para avaliar economicamente a eficiência da aquisição de medicamentos é essencial para garantir que haja medicamentos em quantidade necessária, evitando o desabastecimento e corroborando para o uso racional de medicamentos. Portanto, os objetivos do estudo foram (i) propor indicadores de avaliação de aquisição de medicamentos e (ii) avaliar o resultado econômico na aquisição dos medicamentos do Hospital Estadual Américo Brasiliense (HEAB) por meio dos indicadores propostos.

\section{Métodos}

Realizou-se um estudo descritivo e transversal no Hospital Estadual Américo Brasiliense (HEAB), São Paulo, Brasil. Trata-se de um hospital de média complexidade, integrado ao SUS, com 104 leitos (HEAB, 2016).

Foi avaliado o processo de compra de medicamentos em um período de 12 meses (abril de 2015 a março de 2016). Os pedidos de compra de medicamentos do HEAB são realizados pela farmacêutica responsável da instituição de acordo com prévia análise do estoque e em quantidades necessárias para dois meses de uso.

A frequência de compra para cada medicamento variou de acordo com seu consumo, mês a mês. Em geral, as compras foram realizadas a cada dois meses (média $=0,68 /$ mês, $\mathrm{DP}=0,24)$.

No período do estudo foram adquiridas 560 apresentações de medicamentos, totalizando um gasto de $\mathrm{R} \$ 1.666 .503,69$. As compras foram realizadas segundo regimento da Fundação que administra o Hospital (FAEPA, 2008). Esse regimento foi aprovado pelo tribunal de contas do estado de São Paulo, com um teto de R\$180 mil/ano por unidade comprada, ou seja, se a compra não ultrapassar o teto/ano, são adquiridos por cotação simples e com necessidade mínima de três orçamentos (FAEPA, 2008). 
A coleta de dados foi realizada por meio de pesquisa documental dos relatórios de compra e de consumo de medicamentos referentes aos meses de abril de 2015 a março de 2016, disponibilizados pelo setor de compras do HEAB. Foram coletadas informações como o número de medicamentos adquiridos em cada compra, bem como seus valores unitários e a taxa de consumo mensal em unidades. Os dados foram processados em planilhas do Software Microsoft Excel 2010 para a elaboração da curva ABC e o cálculo dos indicadores de percentual econômico e de razão econômica.

Para a elaboração da curva ABC, utilizaram-se o preço unitário de todas as apresentações compradas e seus respectivos valores de consumo mensal, a fim de calcular o percentual acumulado dos itens (Vecina Neto e Reinhardt Filho 1998). Foram identificadas 72 apresentações que correspondem a $95 \%$ dos gastos ( $R \$ 1.583 .178,50)$, sendo 26 classificados como A (cerca de $20 \%$ dos itens do estoque e cerca de $80 \%$ do valor total do estoque) e 46 como B (cerca de $15 \%$ dos itens do estoque e cerca de $15 \%$ do valor total do estoque). Dessa forma, as 488 apresentações que, segundo a curva ABC, foram classificadas como C (85\% das apresentações) não foram utilizadas na elaboração dos indicadores, visto que correspondem a apenas 15\% dos gastos.

Para o primeiro indicador de avaliação econômica da aquisição de medicamentos, o Percentual Econômico, propôs-se a aplicação do cálculo "percentual de economia entre o menor e o maior preço observados, um indicador de acesso a medicamentos desenvolvido pela Organização Pan-Americana de Saúde juntamente com o Ministério da Saúde OPAS/ MS, cuja fórmula é (Organização Pan-Americana da Saúde/ Organização Mundial da Saúde/Ministério da Saúde 2005; Ferreira-Filho et al. 2010):

[1-(maior preço unitário/menor preço unitário)] x 100.

Considerou-se o "maior preço unitário" o preço médio das aquisições analisadas e o "menor preço unitário" o preço tabelado como preço máximo de venda ao governo (PMVG) pela CMED (Câmara de Regulação do Mercado de Medicamentos), órgão interministerial, monitorado pela ANVISA e responsável por regular o mercado e estabelecer critérios para a definição e o ajuste de preços (CMED, 2016). A tabela CMED utilizada foi referente a março/2016, considerando a alíquota de ICMS de 18\%, referente ao estado de São Paulo (CMED 2016); na ocorrência de mais de um fabricante para o mesmo medicamento, foi considerado o fabricante com menor preço.

Assim, a fórmula adaptada e utilizada nesse estudo foi:

Percentual Econômico = [1-(preço unitário de aquisição médio/ PMVG (MED)] x 100
Dessa forma, um resultado positivo sinalizou uma economia na compra do medicamento, ou seja, o valor pago era menor que o preço tabelado; um resultado negativo indicou prejuízo na compra; e um resultado igual a zero correspondeu a ausência de economia ou prejuízo para a instituição de saúde.

Já o segundo indicador, Razão Econômica, foi baseado na metodologia desenvolvida pela Organização Mundial da Saúde/Ação de Saúde Internacional denominada "razão de preço de medicamento" ("Medicine Price Ratio"), com o objetivo de levantar informações sobre preço, disponibilidade e acessibilidade de determinados medicamentos, pertencentes aos setores públicos e privados (WHO/HAl, 2008), cuja fórmula é (WHO - World Health Organization/ HAl - Health Action International 2008):

Preço médio = preço unitário médio local / preço unitário de referência internacional

Considerou-se como "preço unitário médio local" o preço médio das aquisições analisadas para cada medicamento, replicando-se o cálculo a cada compra, e como "preço unitário de referência internacional" o preço tabelado PMVG pela CMED (CMED 2016), ICMS 18\%.

Dessa forma, a fórmula adaptada e utilizada nesse estudo foi:

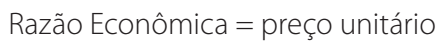

Salienta-se que na presente análise PMVG foi utilizado como referencial por se tratar de um hospital estadual integrado ao SUS. No caso dos indicadores serem utilizados em um serviço privado, recomenda-se que o referencial seja o de preço máximo ao consumidor (PMC) (CMED, 2016).

Para facilitar a replicação dessa metodologia, a WHO/HAl refere o uso da razão como método de comparação entre os preços. Assim, se a razão entre o preço do medicamento coletado no estudo e o preço padronizado internacionalmente for 2, por exemplo, significa que tal medicamento custou o dobro do preço referência.

Além disso, foi calculada a mediana de economia para cada uma das apresentações no período total de estudo (12 meses), bem como a média de economia de todas as apresentações compradas no período.

\section{Resultados}

Foi calculado o resultado econômico da aquisição de 51 apresentações classificadas como A e B, pois para 21 apresentações não havia valores tabelados pela CMED, por serem medicamentos específicos, tais como soluções fisiológicas, 
glicofisiológicas e de ringer. A economia total gerada na aquisição das 51 apresentações, considerando o consumo mensal de cada uma, foi de R\$2.188.120,59.

A mediana da economia total gerada calculada pelo percentual econômico foi de 41,6\% (mínimo =-2008,6\%, máximo $=99,3 \%$, intervalo interquartil (IIQ 25-75\%) $=-15,2-54,9)$ e de 0,6 (mínimo = 0, máximo = 21,1, IIQ 25-75\% = 0,5 - 1,2) calculada pela razão econômica (Tabela 1).
Os medicamentos anfotericina B complexo lipídico frasco-ampola 100 mg 20 ml, ciprofloxacino injetável frasco ou bolsa 200 mg 100 ml e cefepima, clor. injetável foram os medicamentos que mais geraram economia (Tabela 1). Por outro lado, os medicamentos que mais geraram prejuízo foram manitol 20\% injetável, oxacilina injetável frasco-ampola 500 mg e nalbufina, clor., injetável; ao todo, 16 medicamentos geraram prejuízo.

Tabela 1. Apresentações farmacêuticas segundo categoria curva ABC, preço máximo de venda ao governo (PMVG) CMED, valor de aquisição unitário médio de todas as compras, percentual econômico e razão econômica realizada pelo Hospital Estadual de Américo Brasiliense (HEAB), no período de abril de 2015 a março de 2016. Em negrito medicamentos que apresentaram prejuízo no processo de compra

\begin{tabular}{|c|c|c|c|c|c|c|}
\hline Apresentação & $A B C$ & $\begin{array}{l}\text { PMVG - CMED } \\
\text { (R\$) }\end{array}$ & $\begin{array}{l}\text { Valor unitário } \\
\text { médio (R\$) }\end{array}$ & $\begin{array}{l}\text { Economia } \\
\text { ou prejuízo } \\
\text { considerando } \\
\text { consumo médio } \\
\text { mensal (R\$) }\end{array}$ & $\begin{array}{l}\text { Percentual } \\
\text { econômico }\end{array}$ & $\begin{array}{c}\text { Razão } \\
\text { econômica }\end{array}$ \\
\hline $\begin{array}{l}\text { Anfotericina B complexo lipídico } \\
\text { frasco-ampola } 100 \text { mg } 20 \text { ml }\end{array}$ & $A$ & 1871,9 & 13,5 & 112687,1 & 99,3 & 0,01 \\
\hline $\begin{array}{l}\text { Ciprofloxacino inj. Frasco } \\
\text { ou bolsa } 200 \mathrm{mg} 100 \mathrm{ml}\end{array}$ & B & 56,4 & 1,8 & 102344,8 & 96,8 & 0,03 \\
\hline $\begin{array}{l}\text { Cefepima, clor. Injetável } \\
\text { frasco-ampola } 1 \mathrm{~g}\end{array}$ & B & 34,7 & 3,4 & 172976,7 & 90,1 & 0,10 \\
\hline $\begin{array}{l}\text { Meropeném injetável } \\
\text { frasco-ampola } 500 \text { mg }\end{array}$ & A & 73,0 & 7,7 & 668644,8 & 89,4 & 0,11 \\
\hline $\begin{array}{l}\text { Midazolam injetável } \\
\text { ampola } 50 \text { mg } 10 \mathrm{ml}\end{array}$ & B & 15,3 & 2,5 & 45464,3 & 83,4 & 0,17 \\
\hline $\begin{array}{l}\text { Propofol injetável frasco- } \\
\text {-ampola } 200 \text { mg } 20 \text { ml }\end{array}$ & A & 22,5 & 4,0 & 140624,5 & 82,2 & 0,18 \\
\hline $\begin{array}{l}\text { Omeprazol sódico injetável } \\
\text { frasco-ampola } 40 \text { mg }\end{array}$ & B & 20,9 & 3,9 & 100115,1 & 81,1 & 0,19 \\
\hline $\begin{array}{l}\text { Claritromicina injetável } \\
\text { frasco-ampola } 500 \text { mg }\end{array}$ & A & 81,4 & 26,1 & 74444,7 & 67,9 & 0,32 \\
\hline $\begin{array}{l}\text { Lactulose xarope } 667 \\
\mathrm{mg} / \mathrm{ml} \text { frasco } 120 \mathrm{ml}\end{array}$ & B & 18,9 & 6,2 & 16292,0 & 67,4 & 0,33 \\
\hline $\begin{array}{l}\text { Sevoflurano 100\%, } \\
\text { inalatório, frasco } 250 \mathrm{ml}\end{array}$ & A & 716,8 & 262,0 & 80257,9 & 63,5 & 0,37 \\
\hline $\begin{array}{l}\text { Albumina humana sérica } 20 \% \\
\text { injetável frasco-ampola } 50 \text { ml }\end{array}$ & B & 173,6 & 69,0 & 35461,3 & 60,3 & 0,40 \\
\hline $\begin{array}{l}\text { Água para injeção } \\
\text { embalagem } 250 \mathrm{ml}\end{array}$ & B & 4,0 & 1,6 & 21010,9 & 58,9 & 0,41 \\
\hline $\begin{array}{l}\text { Rocurônio, brometo } \\
\text { frasco } 5 \mathrm{ml} 50 \mathrm{mg}\end{array}$ & B & 23,5 & 10,4 & 17337,6 & 55,6 & 0,44 \\
\hline $\begin{array}{l}\text { Piperacilina } 4 \mathrm{~g}+\text { tazobactam } \\
0,5 \mathrm{~g} \text { inj. frasco-ampola }\end{array}$ & A & 61,7 & 28,3 & 160114,6 & 54,1 & 0,46 \\
\hline $\begin{array}{l}\text { Clindamicina, fosfato injetável } \\
\text { amp. } 600 \mathrm{mg} 4 \mathrm{ml}\end{array}$ & B & 8,2 & 3,7 & 22605,5 & 54,1 & 0,46 \\
\hline $\begin{array}{l}\text { Cetoprofeno injetável e.v. } \\
\text { frasco-ampola } 100 \text { mg }\end{array}$ & B & 5,1 & 2,6 & 21499,5 & 49,4 & 0,51 \\
\hline Água para injeção ampola 10 ml & B & 0,3 & 0,1 & 16954,5 & 48,6 & 0,51 \\
\hline $\begin{array}{l}\text { Enoxaparina sódica injetável } \\
\text { seringa } 20 \mathrm{mg} \mathrm{0,2} \mathrm{ml}\end{array}$ & B & 13,4 & 6,9 & 18538,1 & 48,1 & 0,52 \\
\hline
\end{tabular}




\begin{tabular}{|c|c|c|c|c|c|c|}
\hline Apresentação & $A B C$ & $\begin{array}{l}\text { PMVG - CMED } \\
\text { (R\$) }\end{array}$ & $\begin{array}{l}\text { Valor unitário } \\
\text { médio (R\$) }\end{array}$ & $\begin{array}{l}\text { Economia } \\
\text { ou prejuízo } \\
\text { considerando } \\
\text { consumo médio } \\
\text { mensal (R\$) }\end{array}$ & $\begin{array}{l}\text { Percentual } \\
\text { econômico }\end{array}$ & $\begin{array}{c}\text { Razão } \\
\text { econômica }\end{array}$ \\
\hline $\begin{array}{l}\text { Enoxaparina sódica injetável } \\
\text { seringa } 40 \mathrm{mg} \mathrm{0,4} \mathrm{ml}\end{array}$ & A & 25,9 & 13,5 & 94286,6 & 48,0 & 0,52 \\
\hline $\begin{array}{l}\text { Enoxaparina sódica injetável } \\
\text { seringa } 80 \mathrm{mg} \mathrm{0,8} \mathrm{ml}\end{array}$ & A & 51,8 & 27,5 & 49034,6 & 47,0 & 0,53 \\
\hline $\begin{array}{l}\text { Água para injeção } \\
\text { embalagem } 1000 \text { ml }\end{array}$ & $B$ & 6,2 & 3,3 & 8907,3 & 46,7 & 0,53 \\
\hline $\begin{array}{l}\text { Enoxaparina sódica injetável } \\
\text { seringa } 60 \mathrm{mg} \mathrm{0,6} \mathrm{ml}\end{array}$ & A & 38,6 & 20,6 & 36337,5 & 46,6 & 0,53 \\
\hline $\begin{array}{l}\text { Amoxicilina } 1000 \text { mg } \\
\text { + clavulanato } 200 \text { mg } \\
\text { injetável frasco-ampola }\end{array}$ & $B$ & 13,0 & 7,1 & 13811,6 & 45,6 & 0,54 \\
\hline $\begin{array}{l}\text { Polimixina b, sulfato injetável } \\
\text { frasco-ampola } 500.000 \mathrm{UI}\end{array}$ & A & 57,9 & 31,5 & 34469,6 & 45,5 & 0,54 \\
\hline $\begin{array}{l}\text { Vancomicina, clor. Injetável } \\
\text { frasco-ampola } 500 \text { mg }\end{array}$ & A & 9,1 & 5,2 & 23664,2 & 43,0 & 0,57 \\
\hline $\begin{array}{l}\text { Ropivacaína, clor. } 1 \% \\
\text { injetável ampola } 20 \mathrm{ml}\end{array}$ & B & 25,0 & 14,6 & 22610,7 & 41,6 & 0,58 \\
\hline $\begin{array}{l}\text { Anidulafungina, injetável, } \\
\text { frasco-ampola } 100 \text { mg }\end{array}$ & B & 282,2 & 181,1 & 11110,7 & 35,8 & 0,64 \\
\hline $\begin{array}{l}\text { Ceftriaxona sódica injetável } \\
\text { e.v. frasco-ampola } 1 \mathrm{~g}\end{array}$ & $A$ & 5,2 & 3,3 & 23134,2 & 35,3 & 0,65 \\
\hline $\begin{array}{l}\text { Aciclovir injetável frasco- } \\
\text {-ampola } 250 \text { mg }\end{array}$ & B & 44,6 & 30,2 & 6183,6 & 32,2 & 0,68 \\
\hline $\begin{array}{l}\text { Etonogestrel } 68 \text { mg } \\
\text { implante via subdérmica }\end{array}$ & A & 651,9 & 508,0 & 20474,6 & 22,1 & 0,78 \\
\hline $\begin{array}{l}\text { Fentanila, citrato, injetável } \\
\text { frasco-ampola 0,50 mg } 10 \mathrm{ml}\end{array}$ & B & 3,2 & 2,8 & 5782,6 & 12,2 & 0,88 \\
\hline $\begin{array}{l}\text { Morfina, sulfato, injetável } \\
\text { ampola } 10 \mathrm{mg} 1 \mathrm{ml}\end{array}$ & B & 2,2 & 1,9 & 1188,0 & 11,4 & 0,89 \\
\hline $\begin{array}{l}\text { Dipirona sódica injetável } \\
\text { ampola } 1 \mathrm{~g} 2 \mathrm{ml}\end{array}$ & B & 0,4 & 0,4 & 3382,2 & 9,9 & 0,90 \\
\hline $\begin{array}{l}\text { Lidocaína 2\% geleia } \\
\text { esteril seringa } 10 \mathrm{~g}\end{array}$ & A & 11,9 & 10,8 & 9677,4 & 9,1 & 0,91 \\
\hline $\begin{array}{l}\text { Ciprofloxacina + dexametasona } \\
\text { colírio frasco } 5 \mathrm{ml}\end{array}$ & B & 12,1 & 12,4 & 6609,1 & $-2,9$ & 1,03 \\
\hline $\begin{array}{l}\text { Heparina sódica subcutânea } \\
\text { ampola } 5.000 \text { UI 0,25 ml }\end{array}$ & A & 2,9 & 3,2 & 5863,1 & $-9,4$ & 1,09 \\
\hline $\begin{array}{l}\text { Sugamadex injetável frasco- } \\
\text {-ampola } 200 \mathrm{mg} 2 \mathrm{ml}\end{array}$ & B & 221,6 & 250,9 & 1446,7 & $-13,2$ & 1,13 \\
\hline $\begin{array}{l}\text { Bupivacaína, clor. 0,5\% } \\
\text { pesada inj. Amp. } 4 \mathrm{ml}\end{array}$ & B & 5,7 & 6,5 & 510,5 & $-14,4$ & 1,14 \\
\hline $\begin{array}{l}\text { Poliestirenossulfonato } \\
\text { de cálcio pacote } 30 \mathrm{~g}\end{array}$ & B & 13,5 & 15,6 & 747,6 & $-15,9$ & 1,16 \\
\hline $\begin{array}{l}\text { Alprostadil injetável } \\
\text { ampola } 20 \mathrm{mcg}\end{array}$ & B & 50,0 & 58,5 & 404,4 & $-17,0$ & 1,17 \\
\hline $\begin{array}{l}\text { Norepinefrina, hemitartarato } \\
\text { injetável ampola } 8 \mathrm{mg} / 4 \mathrm{ml}\end{array}$ & A & 3,2 & 3,7 & 1594,8 & $-18,5$ & 1,19 \\
\hline $\begin{array}{l}\text { Tigeciclina injetável } \\
\text { frasco-ampola } 50 \mathrm{mg}\end{array}$ & B & 129,5 & 154,3 & 482,7 & $-19,2$ & 1,19 \\
\hline
\end{tabular}




\begin{tabular}{|c|c|c|c|c|c|c|}
\hline Apresentação & $\mathrm{ABC}$ & $\begin{array}{l}\text { PMVG - CMED } \\
\text { (R\$) }\end{array}$ & $\begin{array}{l}\text { Valor unitário } \\
\text { médio (R\$) }\end{array}$ & $\begin{array}{l}\text { Economia } \\
\text { ou prejuízo } \\
\text { considerando } \\
\text { consumo médio } \\
\text { mensal (R\$) }\end{array}$ & $\begin{array}{l}\text { Percentual } \\
\text { econômico }\end{array}$ & $\begin{array}{l}\text { Razão } \\
\text { econômica }\end{array}$ \\
\hline $\begin{array}{l}\text { Cefazolina injetável } \\
\text { frasco-ampola } 1 \mathrm{~g}\end{array}$ & B & 3,3 & 4,0 & 1021,8 & $-20,6$ & 1,21 \\
\hline $\begin{array}{l}\text { Remifentanila, clor, injetável } \\
\text { frasco-ampola } 2 \mathrm{mg}\end{array}$ & A & 26,8 & 35,1 & $-2483,1$ & $-30,9$ & 1,31 \\
\hline Octreotida ampola $1 \mathrm{mg} 1 \mathrm{ml}$ & B & 31,1 & 41,4 & 1235,2 & $-33,1$ & 1,33 \\
\hline $\begin{array}{l}\text { Dobutamina, cloridrato, } \\
\text { injetável ampola } 250 \mathrm{mg} 20 \mathrm{ml}\end{array}$ & B & 14,2 & 19,2 & 100,3 & $-34,9$ & 1,35 \\
\hline $\begin{array}{l}\text { Nicotina adesivo } \\
\text { transdérmico } 21 \mathrm{mg}\end{array}$ & B & 5,0 & 6,8 & 797,1 & $-36,8$ & 1,37 \\
\hline $\begin{array}{l}\text { Pancurônio, brometo, } \\
\text { injetável ampola } 2 \mathrm{ml} 4 \mathrm{mg}\end{array}$ & B & 3,3 & 4,6 & $-583,2$ & $-39,6$ & 1,40 \\
\hline $\begin{array}{l}\text { Nalbufina, clor, injetável } \\
\text { ampola } 10 \mathrm{mg} 1 \mathrm{ml}\end{array}$ & B & 6,9 & 11,0 & $-2510,9$ & $-58,8$ & 1,59 \\
\hline $\begin{array}{l}\text { Oxacilina injetável frasco- } \\
\text {-ampola } 500 \mathrm{mg}\end{array}$ & A & 1,5 & 2,5 & $-6223,0$ & $-66,9$ & 1,67 \\
\hline $\begin{array}{l}\text { Manitol } 20 \% \text { injetável } \\
\text { embalagem rígida } 250 \mathrm{ml}\end{array}$ & B & 0,2 & 4,0 & $-12321,3$ & $-2008,6$ & 21,09 \\
\hline Mediana & & & & & 41,6 & 0,58 \\
\hline Mínimo & & & & & $-2008,6$ & 0,01 \\
\hline Máximo & & & & & 99,3 & 21,09 \\
\hline Intervalo interquartil (25-75\%) & & & & & $-15,2-54,9$ & $0,45-1,15$ \\
\hline
\end{tabular}

ABC: Classificação ABC das apresentações; PMVG - CMED: preço máximo de venda ao governo tabelado pela Câmara de Regulação do Mercado de Medicamentos.

\section{Discussão}

A aquisição de medicamentos deve ser monitorada por indicadores quantitativos e qualitativos, tais como a utilização de avaliações econômicas para a manutenção da qualidade dos serviços de saúde (Santana et al., 2014). No caso dos serviços públicos, os indicadores são um modo de monitorar a razoabilidade e a eficiência dos gastos públicos a fim de atender aos princípios do direito administrativo (Brasil, 1988).

A OMS lista exemplos de ações relacionadas à aquisição de medicamentos que influenciam o preço, a disponibilidade e/ou a acessibilidade. Destacamos a realização de contratos competitivos com transparência de preços, uso de avaliações econômicas em saúde ou comparações internacionais de preços como diretrizes para a fixação de preços de produtos referências e garantia de transparência e monitoramento de preços de qualidade e informação pública (WHO - World Health Organization/ HAl - Health Action International, 2008).

Nesse contexto, a OMS propõe o uso do MPR (Medicine Price Ratio, Razão do Preço do Medicamento) para avaliação da eficiência de aquisição de medicamentos de uma região ou país, utilizando como parâmetro a razão entre o preço unitário local médio pelo preço de referência internacional, em vez do preço de fábrica CMED proposto no presente estudo, com o objetivo de permitir uma comparação entre os países (WHO - World Health Organization/HAl - Health Action International, 2008).

A proposta de comparar o preço de aquisição com o preço de fábrica CMED, tanto por meio do percentual econômico quanto pela razão econômica, se justifica, pois este é o preço máximo permitido para venda a farmácias, drogarias e para entes da administração pública, praticado pelas empresas produtoras ou importadoras do produto e pelas empresas distribuidoras.

Faria et al. (Faria et al., 2005) ressaltam a importância de avaliações econômicas em saúde como auxílio para tomada de decisões que visam à otimização de recursos no sistema de saúde, pois levam em consideração o fator econômico para a escolha de alternativa terapêutica. Avaliações econômicas em saúde compreendem análises comparativas entre ao menos duas tecnologias com relação aos custos e consequências em saúde (Drummond et al., 2005). 
Os indicadores propostos visaram avaliar a eficiência econômica da aquisição de medicamentos, ou seja, as alternativas comparadas correspondem às mesmas especialidades terapêuticas comercializadas por diferentes fornecedores e, portanto, espera-se desempenho equivalente das tecnologias comparadas em termos de eficácia e segurança.

Ademais, evidenciou que o processo de compra diferente do proposto pelo processo licitatório foi eficiente, logo o seu resultado pode ser utilizado como benchmarking funcional para outros processos de compra, incluindo o licitatório (Bittar, 2001).

Portanto, esses indicadores possuem um caráter exclusivamente econômico, analisando se a aquisição de medicamentos foi ou tem sido satisfatória em relação a um parâmetro fixo, como a lista de preços de fábrica de medicamentos, de forma simples e prática e/ou o seu benchmarking.

Ambos os indicadores propostos possuem as características de um indicador de resultado adequado. Eles são de aplicação simples, pois, utilizando apenas cerca de 12\% dos medicamentos adquiridos, são capazes de refletir a eficiência da compra, visto que gerou economia de $\mathrm{R} \$ 2.188 .120,59$. Também, são confiáveis e significativos, visto que utilizam cálculos matemáticos que podem ser replicados a outras situações e avaliam economicamente a aquisição de maneira eficiente. A mais eles são de baixo custo de implantação, específicos, simples e objetivos (Bittar, 2001).

Ambos se mostraram eficientes para avaliar cada compra, pois, nesse caso, viabilizou identificar possíveis prejuízos pontuais gerados na compra de alguns medicamentos de menor economia ou de gastos maiores que o valor tabelado, permitindo ações pontuais para a melhoria da aquisição, tais como contato com fornecedores, gerando histórico de compra e de fornecedores.

O histórico de compras e histórico de fornecedores são dados imprescindíveis para o cumprimento de boas práticas de aquisição na assistência farmacêutica por permitir obtenção de preços menores, agilizar o processo de aquisição e de contratação do licitante (Brasil/Ministério da Saúde, 2006).

É importante destacar que o volume de compras é um fator de grande influência no preço unitário dos medicamentos. Isso ocorre, pois, quanto maior o volume a ser adquirido, maior será o interesse dos fornecedores, aumentando a concorrência entre eles e, consequentemente, levando a um menor preço ofertado. Porque um volume maior pode atrair fornecedores de maior porte que, além de praticarem um preço mais próximo ao do produtor, possuem lucro unitário menor aplicado a uma quantidade maior de produtos (Marin et al., 2003).

Entretanto, isso não explica, por exemplo, o fato de que a compra de alguns medicamentos não gerou economia (percentual econômico $<0$ e razão econômica $>1$ ). Para esses medicamentos, o preço pago na aquisição foi maior que o PMVG tabelado. O prejuízo gerado na compra das apresentações pode ter ocorrido por falhas no processo de negociação de compra que está sendo monitorado. No caso de compras pelo processo de licitação, poderia ser devido a compras não feitas diretamente com o fabricante, nas quais valores de impostos e transporte podem estar embutidos no preço do medicamento ou, ainda, devido a entregas parceladas encarecendo o produto considerando todo o processo de distribuição e atividades agregadas (Marin et al., 2003).

Portanto, os medicamentos que obtiveram percentual econômico positivo ou razão menor que $\underline{1}$ representam as compras que geraram economia, ou seja, as apresentações foram adquiridas por um valor menor que o PMVG tabelado. Já aqueles com percentual econômico negativo ou razão maior ou igual a 1 permitiram uma maior atenção e mudanças de estratégias no próximo processo de compra, tais como compras maiores com entregas parceladas e busca de cadastrar novos fornecedores.

Como limitação do estudo, destacamos que não foi considerado o preço internacional, tendo em vista que muitos dos medicamentos consultados não foram encontrados na base consultada e ainda que, ao utilizar o preço internacional, outras incertezas poderiam ser agregadas à análise como taxa de câmbio.

\section{Considerações finais}

O processo de aquisição do HEAB foi considerado eficiente no período estudado, porque gerou uma economia $41,6 \%$ ( mínimo $=-2008,6 \%$, máximo $=99,3 \%$, intervalo interquartil $($ IIQ 25-75\%) =-15,2-54,9) e de 0,6 ( mínimo = 0, máximo = 21,1, IIQ $25-75 \%=0,5-1,2)$ segundo os indicadores de percentual econômico e razão econômica, respectivamente, considerando como referencial os preços máximo de venda ao governo CMED, o que evidencia que o processo de compra regimentado pelo hospital otimiza os gastos em aproximadamente $60 \%$. Estima-se que se as medidas propostas forem implantadas no HEAB, poderá aumentar a economia gerada e a eficiência no processo de aquisição.

Devido à similaridade entre os resultados dos dois indicadores, qualquer um deles poderia ser aplicado para o monitoramento da economia gerada. No entanto, o indicador razão econômica seria mais bem aceito e implantável pelo serviço devido a sua simplicidade, maior facilidade de cálculo e interpretação do que o indicador percentual econômico.

\section{Referências bibliográficas}

Bittar OJNV. Indicadores de qualidade e quantidade em saúde. RAS. 2001;3(12):21-8.

Brasil. Constituição da República Federativa do Brasil de 1988. Braślia, DF: Diário Oficial da União.

Brasil. Lei no 10.520, de 17 de Junho de 2002: Institui, no âmbito da União, Estados, Distrito Federal e Municípios, nos termos do art. 37, inciso XXI, da Constituição Federal, modalidade de licitação denominada pregão, para aquisição de bens e serviços comuns, e. Diário Oficial da União. 
Brasil. Lei no 8.666, de 21 de Junho de 1993. Regulamenta o art. 37, inciso XXI, da Constituição Federal, institui normas para licitações e contratos da Administração Pública e dá outras providências, 1993.

Brasil. Portaria no 3.916, de 30 de outubro de 1998. [Internet]. Dispõe sobre a Política Nacional de medicamentos. Brasília; 1998 [citado 12 de março de 2015]. Disponível em: http://dtr2001.saude.gov.br/sas/PORTARIAS/ Port2001/GM/GM-696.htm.

Brasil/Ministério da Saúde. Aquisição de medicamentos para assistência farmacêutica no SUS: orientações básicas. Brasília, DF; 2006.

Capucho H. Farmacovigilância hospitalar: processos investigativos em farmacovigilância. Pharm Bras. 2008;12.

Capucho HC, Mastroianni PC, Cuffini S. Farmacovigilância no Brasil: a relação entre polimorfismo de fármacos, efetividade e segurança dos medicamentos. Rev Ciênc Farm Básica Apl. 2008;29:277-83.

CMED. Lista de preços de medicamentos: preço fábrica e preço máximo de venda ao governo. [Internet]. Agência Nacional de Vigilância Sanitária - Secretaria executiva; 2016 [citado 1 de abril de 2016]. Disponível em: http://portal.anvisa.gov.br/cmed.

Conferencia de Expertos. Uso Racional de Medicamentos. Informe de La Conferencia de Expertos, Nairobi, 25-29 de noviembre de 1985. In Ginebra: Organización Mundial de La Salud; 1986. p. 304.

Donabedian A. Evaluating the Quality of Medical Care. Milbank Q. dezembro de 2005;83(4):691-729.

Drummond M, Sculpher M, Claxton K, Stoddart G, Torrance G. Methods for the economic evaluation of health care programmes. New York: Oxford University Press; 2005.

FAEPA. Regulamento de licitação e contrato da fundação de apoio ao ensino, pesquisa e assistência do Hospital das Clínicas da Faculdade de Medicina de Ribeirão Preto da Universidade de São Paulo - FAEPA; 2008.

Faria ACM, Giraud CS, Pereira ML, Baldoni A de O. Estudos Farmacoeconômicos no Brasil: onde estamos? Rev Bras Farm Hosp Serv Saúde. 2005;5(4):13-8.
Ferreira-Filho JCR, Correia GT, Mastroianni PC. Acesso a medicamentos essenciais em farmácias e drogarias do Município de Araraquara. Rev Ciênc Farm Básica Apl. 2010;31(2):177-82.

HEAB. A História do Hospital Estadual Américo Brasiliense [Internet]. Hospital Estatual Américo Brasiliense. 2016 [citado 7 de março de 2017]. Disponível em: http://www.heab.fmrp.usp.br/Home/ Conteudo?ldNoticia $=4$.

Magarinos-Torres R, Osório-de-Castro CGS, Pepe VLE. Critérios e indicadores de resultados para a farmácia hospitalar brasileira utilizando o método Delfos. Cad Saude Publica. 2007;23(8):1791-802.

Marin N, Luiza VL, Osorio-de-Castro CGS, Machado-dos-Santos S. Assistência farmacêutica para gerentes municipais. Rio de Janeiro: Opas/OMS; 2003. p. 373 .

Mastroianni PC, Lucchetta RC. Regulamentação sanitária de medicamentos. Rev Ciênc Farm Básica Apl. 2011;32(1):127-32.

Organização Pan-Americana da Saúde/Organização Mundial da Saúde/ Ministério da Saúde. Avaliação da Assistência Farmacêutica no Brasil. Brasília; 2005. p. 260.

Santana RS, Jesus EMS de, Santos DG dos, Lyra Júnior DP de, Leite SN, Silva WB da. Indicadores da seleção de medicamentos em sistemas de saúde: uma revisão integrativa. Rev Panam Salud Pública. 2014;35(3):228-34.

Secoli SR, Padilha KG, Litvoc J, Maeda ST. Farmacoeconomia: perspectiva emergente no processo de tomada de decisão. Cien Saude Colet. 2005;10:287-96.

Vecina Neto G, Reinhardt Filho W. Gestão de Recursos Materiais e de Medicamentos. São Paulo: Faculdade de Saúde Pública da Universidade de São Paulo; 1998.

WHO - World Health Organization/ HAI - Health Action International. Measuring medicine prices, availability, affordability and price components. 2a ed. Switzerland; 2008. 